\title{
Decay of a square pulse to Sine-Gordon breathers
}

\author{
G. Kälbermann* \\ Soil and Water dept., Faculty of Agriculture, Rehovot 76100, Israel
}

March 28, 2018

\begin{abstract}
We investigate numerically and analytically the existence of thresholds for the production of Sine-Gordon single and multiple breathers from simple initial pulses.

PACS 02.30.Jr, 03.40.Kf, 03.50.-z, 03.65.Ge, 03.75.Lm, 05.45.Yv, 11.10.Lm, 63.20.Pw

*e-mail address: hope@vms.huji.ac.il
\end{abstract}




\section{$1 \quad$ Introduction}

The Sine-Gordon equation arises in physical problems such as one dimensional dislocations [1], long Josephson junctions[2], as well as in the mathematical treatment of constant negative curvature metric spaces[3].

Solitary wave solutions of the Sine-Gordon equation carry a topological winding number q. The solitary waves conserve their properties upon collision and are therefore called topological solitons. The winding number zero sector $q=0$ consists of bound soliton-antisoliton

solutions, the breathers, unbound soliton-antisoliton pairs[1], and phonons. The latter are small amplitude solutions, corresponding to the Klein-Gordon linear limit of the equation. The $q=1$ sector solitary wave is the shelf or kink soliton.

The Sine-Gordon equation is one of a gallery of nonlinear equations amenable to treatment by the Inverse Scattering Transform (IST) method. The IST produces soliton solutions based on scattering data of Dirac-like equations for a potential connected to the sought solution. The wave functions and the scattering data determine the potential - hence the name inverse scattering transform - through the integral equations named after Gelfand-Levitan and Marchenko[4].

The power of the IST method lies in the mapping of a nonlinear problem to an eigenvalue Sturm-Liouville problem. We recently used the IST method to treat the decay of distorted kinks to wobble states and phonons. [5] In the present work we investigate the decay of a square pulse to single and multiple breathers and phonons. The IST technique determines the constraints on the parameters of an initial pulse for it to decay into breathers and phonons. These constraints are satisfied at the location of a pole in the transmission amplitude of the IST scattering problem.

The decay of simple pulses to breathers was addressed some time ago by Kivshar and coworkers [6, 7]. Kivshar et al. [6, 7] studied the creation of breathers in long Josephson junctions and easy plane ferromagnets with an inhomogeneous Sine-Gordon equation. With a specific choice of the inhomogeneous source term and under the approximation of a negligible initial field configuration, the Zakharov-Shabat (ZS) equations[8, 9] are simplified considerably and an analytical solution of Kaup[10] could be implemented. In the present work we will focus on the homogeneous Sine-Gordon equation with a non negligible initial pulse.

In section 2 we treat the decay of square pulse analytically using the AKNS[9] version of the Zakharov-Shabat[8] equations. The results are compared to a full numerical treatment of the equations with a more realistic pulse. Section 3 provides numerical support to the analytical results of section 2. Conclusions are drawn in section 4 .

\section{Decay of a square pulse in the IST}

The Sine-Gordon equation is usually expressed in two ways. In space-time coordinates

$$
\frac{\partial^{2} u}{\partial T^{2}}-\frac{\partial^{2} u}{\partial X^{2}}+\sin (u)=0
$$


and in light-cone coordinates $x=\frac{1}{2}(X+T) t=\frac{1}{2}(X-T)$ with unit speed of light $c=1$,

$$
\frac{\partial}{\partial t}\left(\frac{\partial u}{\partial x}\right)-\sin (u)=0
$$

The Klein-Gordon equation for a particle of unit mass is obtained by linearizing Eqs. $(1,2)$. Eq.(1) can be derived from the lagrangian

$$
\mathcal{L}=\int d X\left(\left(\frac{\partial u}{\partial T}\right)^{2}-\left(\frac{\partial u}{\partial X}\right)^{2}+(\cos (u)-1)\right)
$$

possesing a conserved energy

$$
\mathcal{E}=\int d X\left[\left(\frac{\partial u}{\partial T}\right)^{2}+\left(\frac{\partial u}{\partial X}\right)^{2}-(\cos (u)-1)\right] .
$$

The IST method uses solutions of the Sine-Gordon and other nonlinear equations as a potential in a linear scattering problem of an anciliary wave function. The scattering problem is split into a time independent set of equations and a time evolution set. For the space-time formulation of the Sine-Gordon equation the equations are [9]

$$
\begin{aligned}
v_{1, X} & =-i\left(\frac{\xi}{2}-\frac{\cos (u)}{8 \xi}\right) v_{1}+\left(\frac{i}{8 \xi} \sin (u)-\frac{1}{4}\left[\frac{\partial u}{\partial X}+\frac{\partial u}{\partial T}\right]\right) v_{2}, \\
v_{2, X} & =i\left(\frac{\xi}{2}-\frac{\cos (u)}{8 \xi}\right) v_{2}+\left(\frac{i}{8 \xi} \sin (u)+\frac{1}{4}\left[\frac{\partial u}{\partial X}+\frac{\partial u}{\partial T}\right]\right) v_{1}, \\
v_{1, T} & =-i\left(\frac{\xi}{2}+\frac{\cos (u)}{8 \xi}\right) v_{1}-\left(\frac{i}{8 \xi} \sin (u)+\frac{1}{4}\left[\frac{\partial u}{\partial X}+\frac{\partial u}{\partial T}\right]\right) v_{2}, \\
v_{2, T} & =i\left(\frac{\xi}{2}+\frac{\cos (u)}{8 \xi}\right) v_{2}-\left(\frac{i}{8 \xi} \sin (u)-\frac{1}{4}\left[\frac{\partial u}{\partial X}+\frac{\partial u}{\partial T}\right]\right) v_{1}
\end{aligned}
$$

where $\xi$ is a complex spectral parameter.

In eqs.(5), the $X, T$ indices denote partial derivatives with respect to the physical coordinates. Consistency between the set of the first two equations and the second two equations in eq.(5) with a time independent $\xi$ yields the Sine-Gordon equation for $u(x, t)$. The solitons of the Sine-Gordon equation correspond to the bound state sector of the spectrum of the AKNS equations[4]. These bound states appear as poles in the transmission coefficients.

Two sets of independent solutions of the AKNS[9, 4] equations are defined. The first set $\phi=\left(\begin{array}{l}v_{1} \\ v_{2}\end{array}\right) \quad \bar{\phi}=\left(\begin{array}{c}\bar{v}_{1} \\ \bar{v}_{2}\end{array}\right)$

with boundary conditions at $X \rightarrow-\infty$

$$
\phi \rightarrow\left(\begin{array}{l}
1 \\
0
\end{array}\right) e^{-i \eta(X, T)}
$$




$$
\begin{aligned}
\bar{\phi} & \rightarrow\left(\begin{array}{c}
0 \\
-1
\end{array}\right) e^{i \eta(X, T)}, \\
\eta(X, T) & =\left(\frac{\xi}{2}-\frac{1}{8 \xi}\right) X+\left(\frac{\xi}{2}+\frac{1}{8 \xi}\right) T .
\end{aligned}
$$

The second set $\psi=\left(\begin{array}{c}v_{1} \\ v_{2}\end{array}\right) \quad \bar{\psi}=\left(\begin{array}{c}\bar{v}_{1} \\ \bar{v}_{2}\end{array}\right)$

with boundary conditions at $X \rightarrow \infty$

$$
\begin{gathered}
\psi \rightarrow\left(\begin{array}{l}
0 \\
1
\end{array}\right) e^{i \eta(X, T)}, \\
\bar{\psi} \rightarrow\left(\begin{array}{l}
1 \\
0
\end{array}\right) e^{-i \eta(X, T)} .
\end{gathered}
$$

The two sets are connected by the scattering amplitudes $a, \bar{a}, b, \bar{b}$

$$
\begin{aligned}
\phi & =a(\xi) \bar{\psi}+b(\xi) \psi \\
\bar{\phi} & =-\bar{a}(\xi) \psi+\bar{b}(\xi) \bar{\psi}
\end{aligned}
$$

Poles in the transmission amplitude $a(\xi)$ with $\operatorname{Im}(\xi)>0$ generate the solitary waves.

The problem of an initial square pulse can be solved analytically. The analytical solution can then be compared to the solution of eqs.(5) for more realistic pulses.

Consider a static square pulse

$$
\begin{aligned}
u(X, T) & =A \Theta(X) \Theta(w-X), \\
\left.\frac{\partial u(x, T)}{\partial T}\right|_{T=0} & =0, \\
u_{X} & =A(\delta(X)-\delta(w-X)),
\end{aligned}
$$

where $\Theta$ denotes the Heaviside step function, $w$ is the width of the pulse and $A$ its height, and $\delta$ represents the Dirac $\delta$ function. The initial pulse is initially at its maximal (minimal) value.

For $\phi$, the solutions of eq.(5) are

$$
\begin{aligned}
& v_{1}=\Theta(-x) e^{-i \eta(X, 0)}+\Theta(x) \Theta(w-x)\left(B e^{i \beta X}+C e^{-i \beta X}\right)+\Theta(x-w) F e^{-i \eta(X, 0)}, \\
& v_{2}=\Theta(x) \Theta(w-x)\left(D e^{i \beta X}+E e^{-i \beta X}\right)+\Theta(x-w) G e^{i \eta(X, 0)}
\end{aligned}
$$


where $B, C, D, E, F, G$ are $\xi$ dependent constant complex amplitudes and $\eta(X, 0)$ is defined in eq.(6). These amplitudes are determined by inserting eq.(10) in eq.(5) and using $\Theta(0)=\frac{1}{2}$

$$
\begin{aligned}
B & =\frac{2 \delta \epsilon-\left(1-\delta^{2}\right)(\mu-\beta)}{2 \beta\left(1+\delta^{2}\right)} \\
C & =\frac{-2 \delta \epsilon+\left(1-\delta^{2}\right)(\mu+\beta)}{2 \beta\left(1+\delta^{2}\right)} \\
D & =\frac{\beta+\mu}{\epsilon} B \\
E & =\frac{-\beta+\mu}{\epsilon} C \\
F & =\frac{P}{1+\delta^{2}} e^{i \eta(w, 0)} \\
P & =e^{i \beta w} B\left(1-\delta^{2}+2 \delta \frac{\beta+\mu}{\epsilon}\right)+e^{-i \beta w} C\left(1-\delta^{2}+2 \delta \frac{\mu-\beta}{\epsilon}\right) \\
G & =\left(e^{i \beta w} D\left[1-\delta^{2}-2 \delta \frac{\epsilon}{\beta+\mu}\right]+e^{-i \beta w} E\left[1-\delta^{2}-2 \delta \frac{\epsilon}{-\beta+\mu}\right]\right) e^{i \eta(w, 0)} /\left(1+\delta^{2}\right) \\
\mu & =\frac{\xi}{2}-\frac{\cos (A)}{8 \xi}, \\
\epsilon & =\frac{\sin (A)}{8 \xi}, \\
\beta & =\sqrt{\mu^{2}+\epsilon^{2}} \\
\gamma & =\frac{A}{8} .
\end{aligned}
$$

Poles in the transmission amplitude correspond to zeroes of the amplitude a in eq.(8), and to the vanishing of the amplitude $P$. The location of the poles is consequently determined by the implicit equation

$$
P=e^{i \beta w} B\left(1-\delta^{2}+2 \delta \frac{\beta+\mu}{\epsilon}\right)+e^{-i \beta w} C\left(1-\delta^{2}+2 \delta \frac{-\beta+\mu}{\epsilon}\right)=0 .
$$

Single breather poles appear in pairs at $\xi= \pm \alpha+i \beta$, with $\beta \geq 0$ to insure the convergence of the Neumann series of the Volterra integrals of the IST.[4], For a single breather at rest the spectral parameter obeys

$$
|\xi|^{2}=\alpha^{2}+\beta^{2}=\frac{1}{4}
$$

An initially static pulse can decay to two breathers also. Momentum conservation requires the breathers to be emitted back to back with opposite velocity. A new parameter enters the 
problem, the velocity of the breathers. For a breather in motion with velocity $v$ the constraint of eq.(13) changes. It is easier to find the modified constraint in the light cone formulation. A Lorentz boost of a breather (a scalar field) amounts to a Lorentz transformation of the spacetime arguments. In light-cone coordinates with unit speed of light, a boost with velocity $V$ in the positive $X$ direction transforms the light cone coordinates

$$
\begin{aligned}
x & \rightarrow \sqrt{\frac{1-v}{1+v}} x, \\
t & \rightarrow \sqrt{\frac{1+v}{1-v}} t .
\end{aligned}
$$

Inspection of the spatial set of the Zakharov-Shabat equations in light-cone coordinates for the Sine-Gordon case[9]

$$
\begin{aligned}
& v_{1, x}=-i \xi v_{1}-\frac{1}{2} u_{x} v_{2} \\
& v_{2, x}=i \xi v_{2}+\frac{1}{2} u_{x} v_{1}
\end{aligned}
$$

shows that the spectral parameter for a moving breather transforms as $\xi \rightarrow \sqrt{\frac{1+v}{1-v}} \xi$. The constraint of eq.(13) now reads $\alpha^{2}+\beta^{2}=\frac{1-v}{4(1+v)}$. As $v \rightarrow 1$, one pair of poles moves towards the real $\xi$ axis while the other pair is pushed up to infinity. We here focus on the single breather production case and the double breather production is dealt with numerically by integrating the Sine-Gordon equation(see section 3). The analytical treatment of the double breather case by means of the Hirota method[11] will be addressed in a future work. For the three breather production from a stationary pulse, one of the breathers remains at the location of the initial pulse. Therefore, three breather production can be treated as the single breather case.

Eq.(12) was derived for the sharp edge square pulse of eq.(9). This pulse has infinite energy as may be seen by inserting eq.(12) in eq.(4), due to the $\delta$ function discontinuities at $X=0, \pm \frac{1}{2} w$. A more realistic profile that still brings about the features of the square profile consists in the addition of lateral wings, continuous with the square pulse and its derivative at its edges. The modified initial pulse (centered at the origin for convenience) reads

$$
u(X, T=0)=A\left(e^{-z_{1}^{2}} \Theta\left(-\frac{1}{2} w-X\right)+\Theta\left(X+\frac{1}{2} w\right) \Theta\left(\frac{1}{2} w-X\right)+e^{-z_{2}^{2}} \Theta\left(X-\frac{1}{2} w\right)\right)
$$




$$
\begin{aligned}
& z_{1}=\frac{X+\frac{1}{2} w}{\delta w}, \\
& z_{2}=\frac{X-\frac{1}{2} w}{\delta w},
\end{aligned}
$$

with $\delta w$, a width parameter for the wings.

For eq.(16) we could not provide analytical solutions. Eqs.(5) were therefore solved numerically in order to verify the predictions of eq.(12). Eqs.(5) are firstly simplified by the substitution

$$
\begin{aligned}
v_{1} & \rightarrow v_{1} e^{-i \rho(X)} \\
v_{2} & \rightarrow v_{2} e^{i \rho(X)} \\
\rho(X) & =\frac{1}{2}\left(\xi X-\frac{\int \cos (u(X)) d X}{8 \xi}\right) .
\end{aligned}
$$

The spatial set of the AKNS equations(5) now becomes

$$
\begin{aligned}
& v_{1, X}=\left(\frac{i}{8 \xi} \sin (u)-\frac{1}{4}\left[\frac{\partial u}{\partial X}+\frac{\partial u}{\partial T}\right]\right) v_{2} e^{2 i \rho(X)}, \\
& v_{2, X}=\left(\frac{i}{8 \xi} \sin (u)+\frac{1}{4}\left[\frac{\partial u}{\partial X}+\frac{\partial u}{\partial T}\right]\right) v_{1} e^{-2 i \rho(X)},
\end{aligned}
$$

with boundary conditions at $X \rightarrow-\infty, v_{1} \rightarrow 1, v_{2} \rightarrow 0$.

We solved eqs.(18) using a fourth order Runge-Kutta method with double precision in order to achieve a maximal error of $1 \%$. The spatial grid was fixed at $\delta X=.0002$ with $-10<X<10$. Poles in the transmission matrix were located by searching for $D=$ abs $\left(v_{1}(\rightarrow \infty)\right)<10^{-5}$. For the wings width parameter we took $\delta w=0.05$, a small enough value to compare to the analytical results of the square pulse above.

Figure 1 shows area plots for breathers production. The single breather regions were found using the analytical formula of eq.(12), curve labeled AKNS analytical, solutions of eq.(18), curve labeled AKNS numerical, and integration of the Sine-Gordon equation, curve labeled Sine-Gordon numerical. The thresholds for two breathers were obtained from the Sine-Gordon equation integration, while three breather thresholds followed from both the AKNS numerical treatment of eq.(18) and the square pulse formula of eq.(12). The curve labeled Sine-Gordon integration does not match the AKNS prediction. This is due to the fact that the observability of the breathers demands a minimal amplitude for them such that they do not blend into the phonon continuum. The thresholds found by the AKNS equations correspond to $\xi_{\text {thresh }}=0.5+i 0$, hence a vanishing breather height. The SineGordon integration curve was drawn for breather amplitudes corresponding to a pole at $\xi=0.499+i 0.0316$ at which the breather becomes clearly visible. We searched for poles in the parameter region $0<A<10,0.5<w<10$, so that $\delta w$ stayed reasonably smaller than the width of the pulse. 


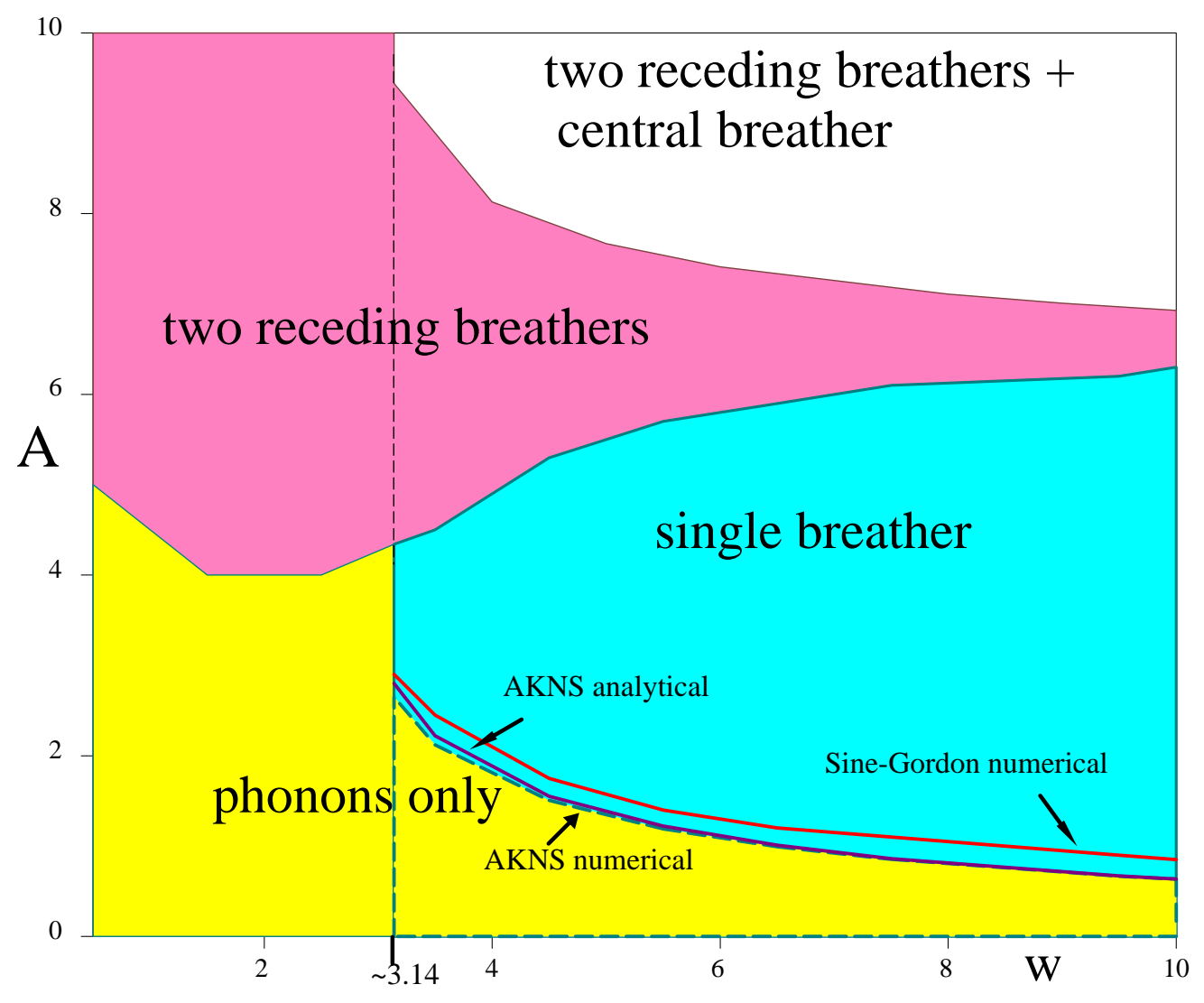

Figure 1: Area plot for the parameter space leading to the production of Sine-Gordon breathers 

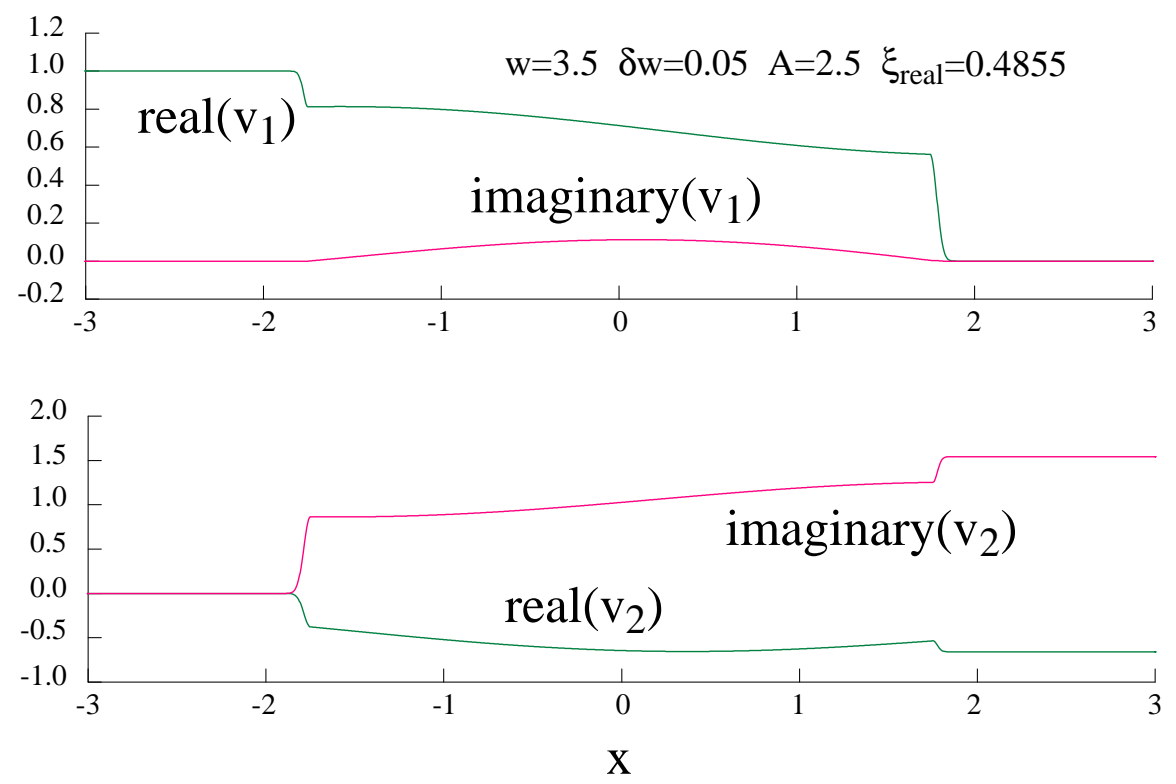

Figure 2: $v_{1}$ and $v_{2}$ of eq.(18) as a function of distance for a value of $\xi$ correponding to a pole in the transmission matrix.

The agreement between the square pulse predictions and the full AKNS treatment is satisfactory. From the plot we see that there is no single or triple breather production unless $w>\approx \pi$. Figure 2 depicts a typical solution of eqs.(18) at a value of the spectral parameter corresponding to a pole. The wave functions are quite constant in each region as in the ansatz of eq.(10) corresponding to the square pulse.

In the next section we depict the various breather production thresholds, and compare the breather profile to the theoretical expression.

\section{Decay of a square pulse, numerical simulations}

The numerical integration of the Sine-Gordon equation, was performed implementing the numerical code described in [5]. The spatial and temporal steps needed to achieve $1 \%$ accuracy in the final energy were $d x=0.02, d t=0.01$. The spatial extent of the integration region was taken to be $X_{\max }=600$, with vanishing fields at infinity. In the examples depicted below we used $\delta w=0.05$.

In section 2 we found two thresholds for single breather production from a square pulse. The width threshold reads $w_{\text {thresh }} \approx \pi$. The height threshold can be obtained from the curve in figure 1. Figure 3 depicts a typical case below the width and height thresholds. A trail of phonons emanating from the origin is clearly observed, as well as the remnant of the original profile. The angular frequency of the oscillation of the central peak is greater than the upper bound for the breather pole $\alpha=\frac{\pi}{\mathbf{T}}=0.523>0.5$, with $\mathbf{T}$, the oscillation period. Moreover, 

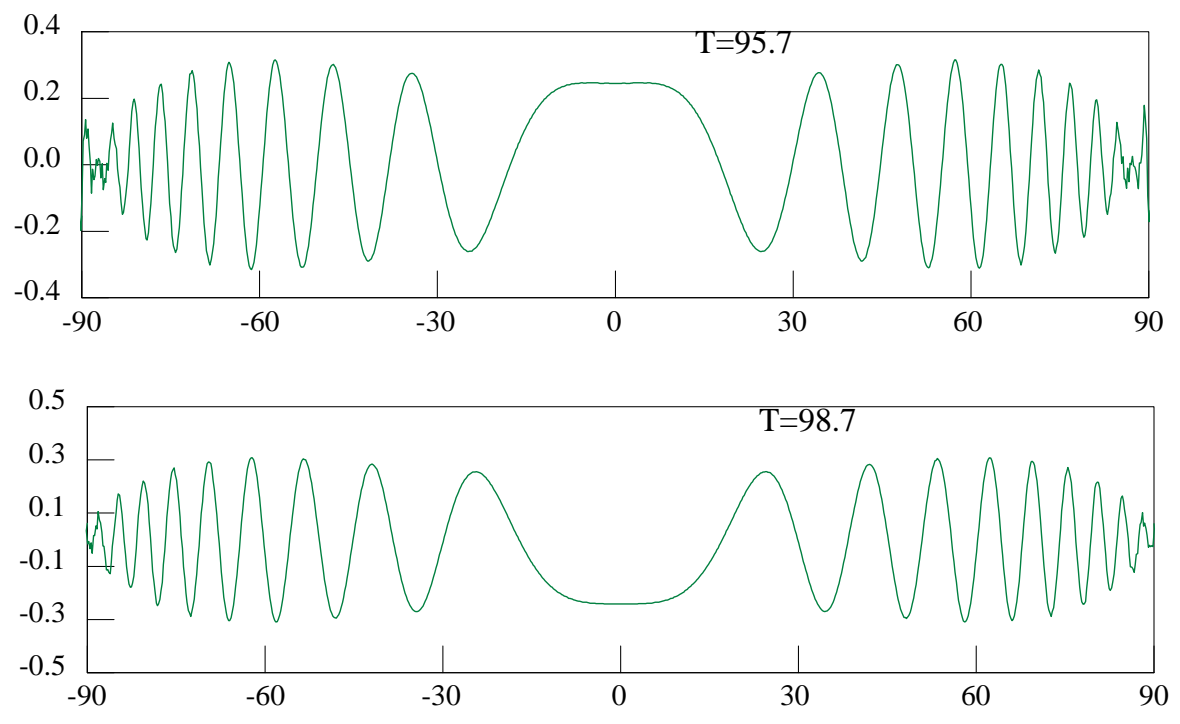

Figure 3: Sine-Gordon solution for an initial pulse with $A=2.5 w=2.5$, below the width and height thresholds.
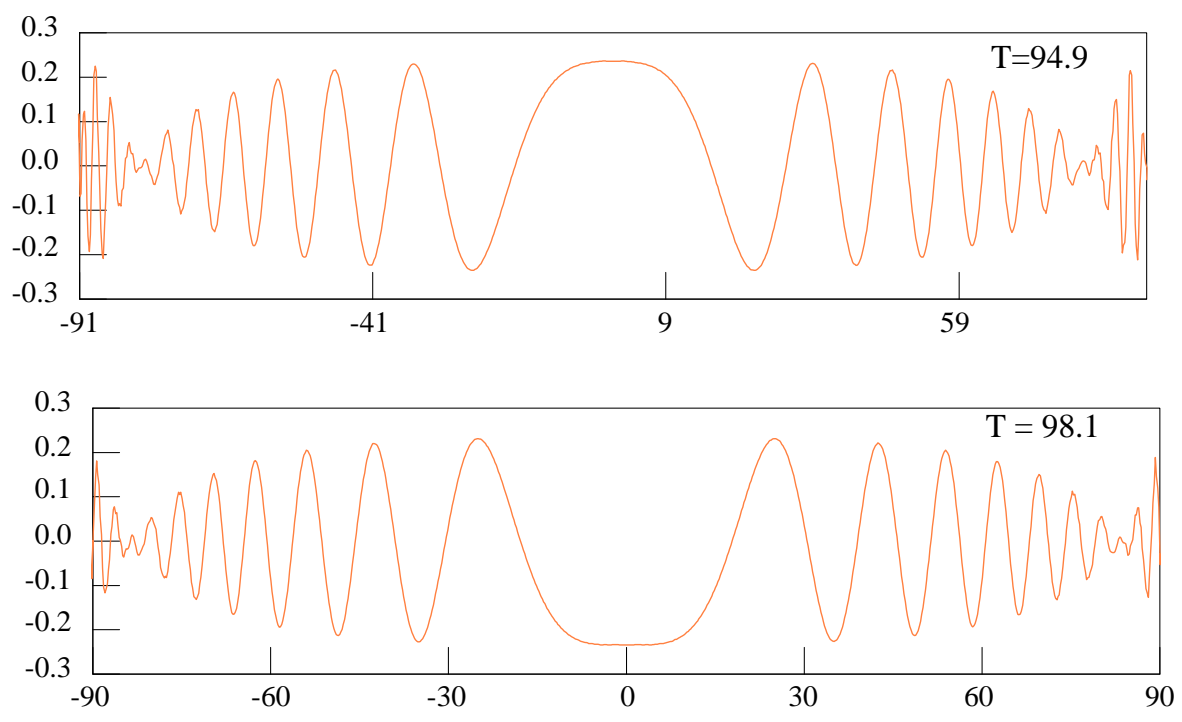

Figure 4: Sine-Gordon solution for an initial pulse with $A=1.5 w=3.5$, above the width threshold and below the height threshold. 


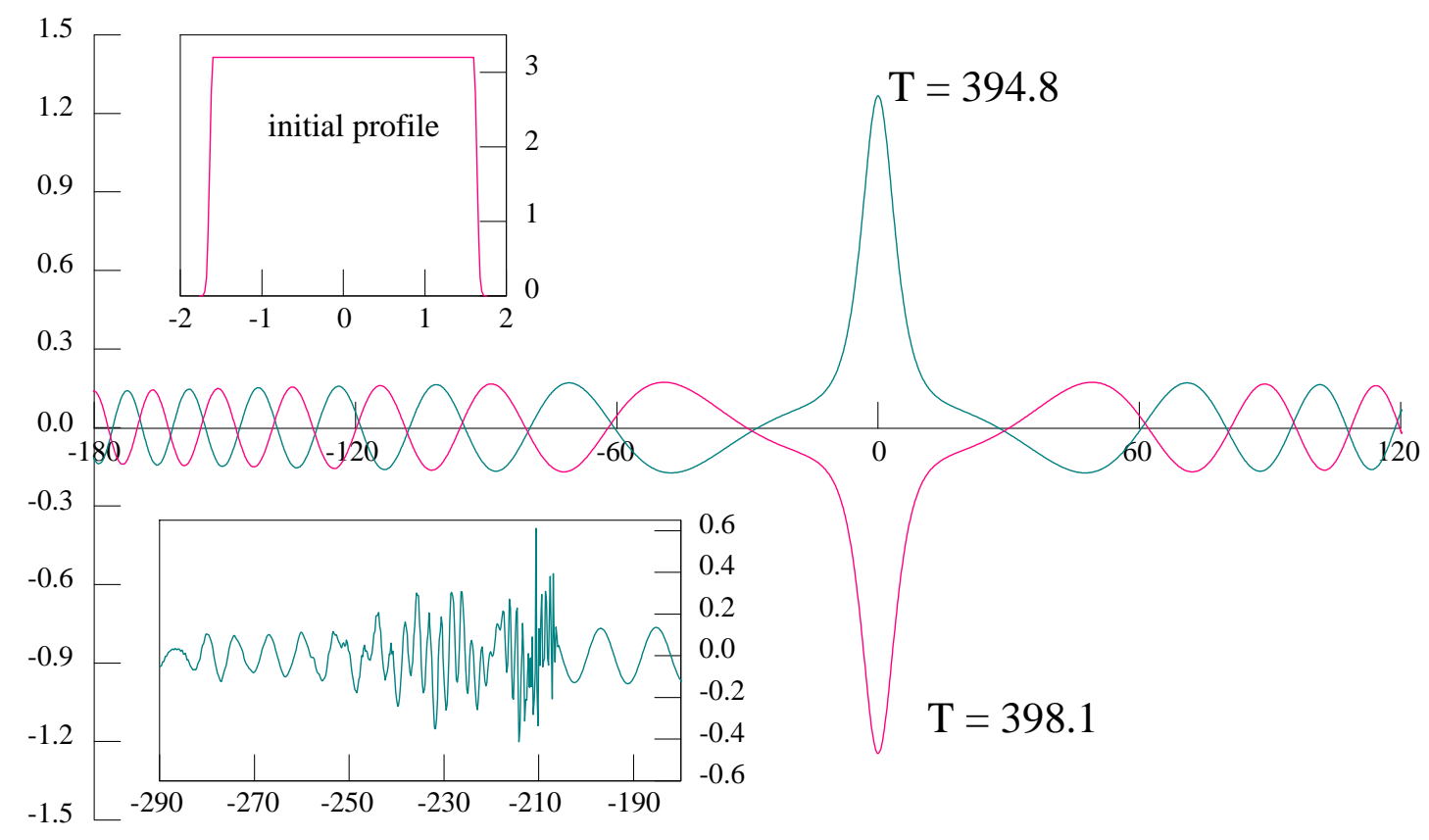

Figure 5: Sine-Gordon solution for an initial pulse with $A=3.2 w=3.2$, above the width and height thresholds.

the central peak does not have the shape of a breather. Figure 4 depicts a case below the height threshold and above the width threshold. Figure 5 shows a case above both width and height thresholds. In figures 3 and 4 only phonons are seen to emanate from the central region, while figure 5 shows a prominent peak centered at the origin and a small trail of phonons in both directions. From the graph of figure 5 the oscillation period is found to be $\mathbf{T}=6.6$. Using $\xi=\alpha+i \beta$, with $\beta=\sqrt{0.25-\beta^{2}}$, and $\alpha=\frac{\pi}{\mathbf{T}}$, we can obtain the location of the pole and evaluate eq.(12) with the amplitude and the height of the pulse as in figure 5 . With these data, eq.(12) yields $P=210^{-3}$, a value close to zero, as compared to $P \approx 1$ for $\xi$ far from a pole location.

We can now compare the shape of the central peak in figure 5, presumed to be a breather, with the theoretical expression

$$
\begin{aligned}
u(X, T) & =4 \tan ^{-1}\left(\frac{\frac{\beta}{\alpha} U}{V}\right), \\
U & =\cos \left(2 \alpha\left(T+T_{0}\right)\right), \\
V & =\cosh (2 \beta X) .
\end{aligned}
$$

This correspondence is depicted in figure 6. Except for small phonon contributions, the profiles fit nicely. The central peak is a breather. 


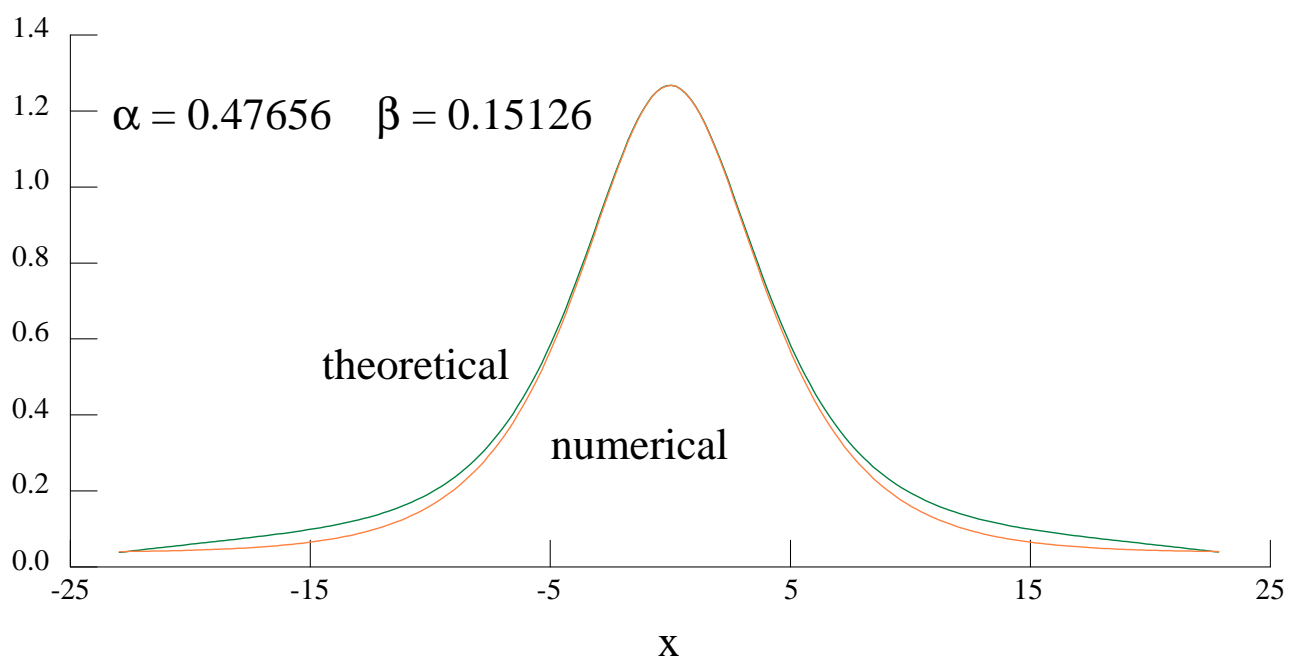

Figure 6: Central peak of the Sine-Gordon solution of figure 5 compared to eq.(19).

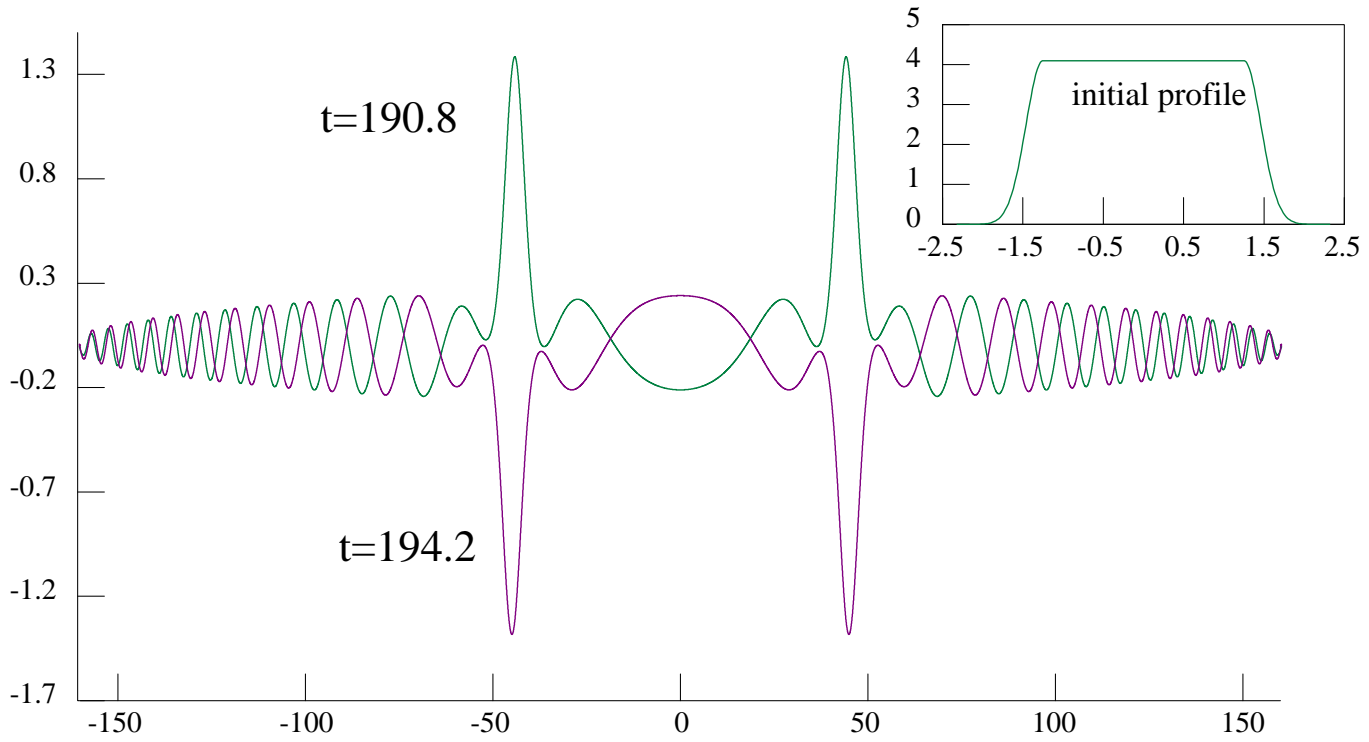

Figure 7: Sine-Gordon equation solution for an initial pulse with $A=4.1, w=2.5$, below the single breather width threshold. 


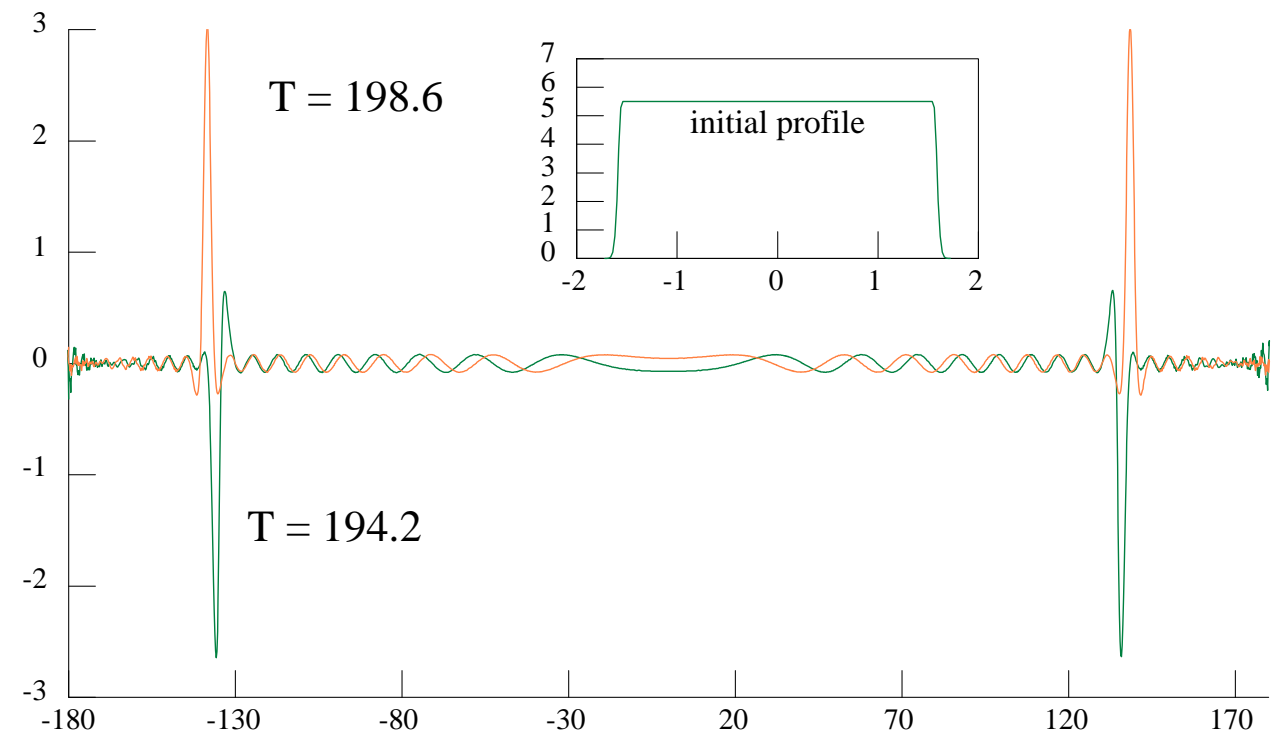

Figure 8: Sine-Gordon equation solution for an initial pulse with $A=5.5, w=3.1$, above the single breather width threshold

Figures 7 and 8 show numerical simulations for the two breather region of figure 1. A pair of back to back breathers receding from the center is observed.

Figure 9 shows a three breather situation.

\section{Conclusions}

In the present investigation we considered the decay of an initially nonvanishing static square pulse. The results complement those of Kivshar et al.[6, 7]. However, contrarily to Kivshar et al.[7] we could not find any case for which a static square pulse leads to soliton-antisoliton pair production regardless of its width, height or energy. A different kind of initial pulse or a time dependent profile is apparently needed.

Among the rich structure of the decaying pulse, the phonon spectrum certainly deserves attention. We found that in general the main features of the spectrum can be reproduced with the long time analytical expressions of Segur and Ablowitz[13], however, the fine details do not match existing expressions in the literature.

We only touched upon the double breather problem and will deal with it in the future.

We conclude with a remark concerning the experimental relevance of the present work. The initial pulse configuration we chose may be realized experimentally in long Josephson junctions of regular or high $T_{c}$ superconductors by injecting two oppositely directed bias currents at a distance $w$ corresponding to the pulse width. By regulating the current intensity and the distance between injection points a well defined breather with a fixed oscillation period may be produced, with possible technological implications. 


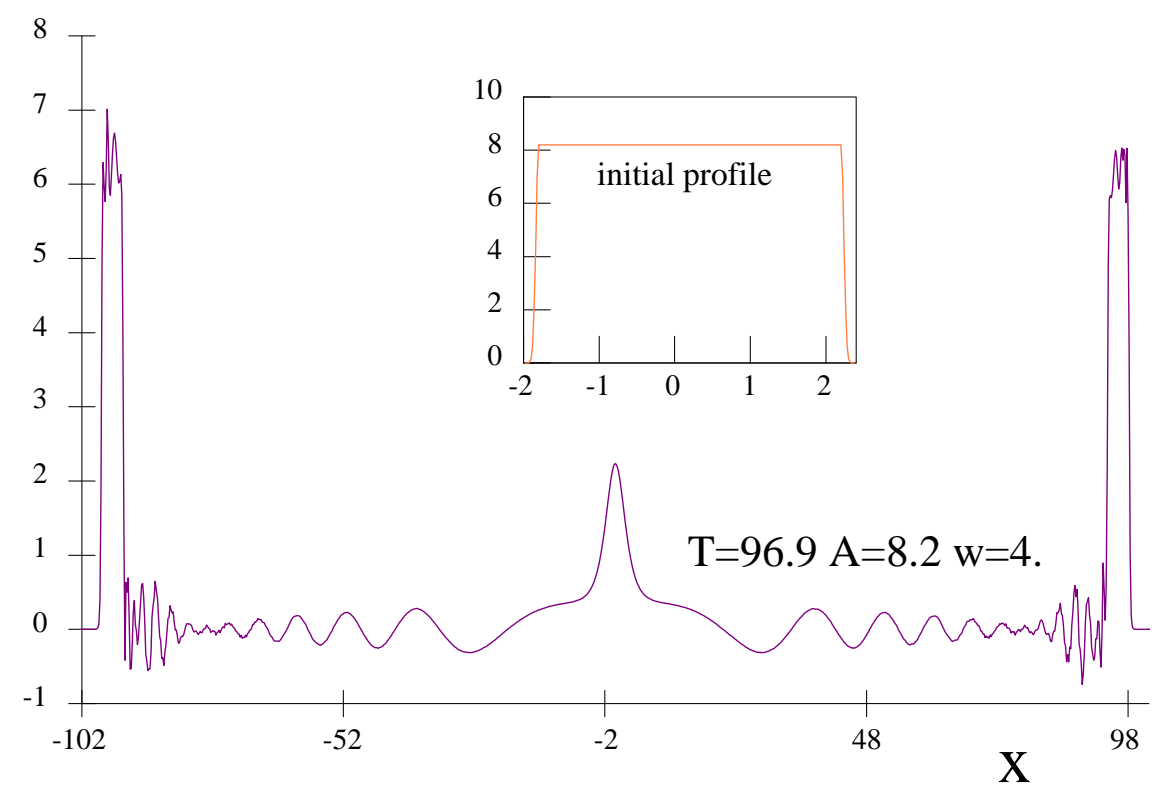

Figure 9: Sine-Gordon equation solution for an initial pulse with $A=8.2, w=4$

\section{References}

[1] G. L. Lamb, Jr., Elements of Soliton theory Wiley Interscience, New York (1980).

[2] M. Remoissenet, Waves called solitons : concepts and experiments , Springer Verlag, Berlin (1996)

[3] L.P. Eisenhart, A Treatise on the Differential Geometry of Curves and Surfaces, Dover, New York (1960).

[4] M. J. Ablowitz and H. Segur Solitons and the Inverse Scattering Transform, SIAM, Philadelphia (1981).

[5] G. Kälbermann, Jour. of Phys. A 37, 11603 (2004).

[6] Yu. S. Kivshar and B. A. Malomed, Sov. Phys. Solid State 31, 293 (1989); Sov. Phys JETP 68, 421 (1989).

[7] Yu. S. Kivshar, B. A. Malomed, Z. Fei and L. Vazquez, Phys. Rev. B42, 1098 (1991).

[8] V. E. Zakharov and P. B. Shabat, Sov. Phys. JETP 3462 (1972).

[9] M. J. Ablowitz, D. J. Kaup, A. C. Newel and H. Segur, Phys. Rev. Lett. 30, 1262 (1973). 
[10] D. J. Kaup Stud. Appl. Math. 54, 165 (1975).

[11] R. Hirota, J. Phys. Soc. Jap. 33, 1459 (1972).

[12] H. Segur, J. Math. Phys. 24, 1439 (1983).

[13] H. Segur and M. J. Ablowitz, Physica 3D, 165 (1981). 